\title{
ON THE TORQUES DUE TO TIDAL FRICTION OF THE OCEANS AND ADJACENT SEAS
}

\author{
P. BROSCHE \\ Astronomisches Rechen-Institut, Heidelberg \\ and \\ J. SUUNDERMANN \\ University of Hamburg
}

\begin{abstract}
The torques of the frictional forces on the bottoms of the seas are calculated in an approximate inertial system and not in the corotating system used hitherto. In this way, the loss of rotational energy of the Earth - corresponding to the mean torque - can be compared with the value obtained from astronomical observations. The calculations are based on velocities which are computed by numerical integrations of the hydrodynamical equations. Initial results are presented for the North Sea, the Bering Sea and rough models of theworld ocean.
\end{abstract}

We now have good evidence that the concept of tidal evolution of the Earth-Moon system is correct for at least the last few hundred million years. However, the question of the geophysical process itself, which is the heart of the subject, cannot yet be considered as solved.

If we focus our attention on the tides of the hydrosphere, we are confronted with the facts that the schematic equilibrium model is unable to serve even as a first approximation and that the empirical data of the real tidal motions are sporadic and too unreliable for the largest part of the oceans and seas. However, recently it has been possible to obtain reasonable theoretical values by the use of hydrodynamicalnumerical models. The introduction of these values is one of our aims.

First we were interested in obtaining the effect of the water on the solid Earth. This interaction between the water and the ground is described by an empirical formula where the frictional force is proportional to the square of the velocity of the water along the ground. The usual application of this formula gives an energy dissipation proportional to the cube of the velocity and this dissipation - integrated over the whole area of the oceans and seas - is compared with the astronomical observed decrease of the rotational energy of the Earth. We now want to criticize this procedure because the astronomical value is referred to an inertial system, while the water effect is referred to a co-rotating system, since the line element in this case is derived from the relative velocity of the water. In order to obtain the net effect on the rotational energy or the equivalent torque, only the east-west components of the frictional forces are to be multiplied with the inertial velocities - for the energy dissipation - or with the corresponding moment arm from the axis - for the torque.

The situation becomes clearer if we not only consider the effect on the Earth, but also the total balance of angular momentum and energy of the acting medium, the water. Since the secular changes of these properties of the water itself are very small compared to the transfer through the water, the time averages of these balances must 
vanish. In the case of angular momentum we can state, first, that there is no microscopic sink or source and therefore the balance consists simply of the two opposite torques of the Moon on the water and the solid Earth on the water. So the calculation of the latter is sufficient. In the case of the energy, the mean dissipation of the kinetic energy of the water will also vanish, but there are more terms necessary to describe this process. The formula for the energy dissipation in the inertial system is,

$$
\dot{E}=\sum m \cdot \mathbf{v} \cdot \mathbf{a}
$$

(where $m$ is the mass, $\mathbf{v}$, the velocity, and a, the acceleration of a water element). The velocity can be expressed as a sum of rotational and relative velocity and the acceleration as a sum of centrifugal, Coriolis and relative acceleration. While it can be shownat least within the limitations of our model - that only the relative acceleration must be taken into account, it is essential that both the relative and the rotational velocities be considered. Then, with respect to the different forces on the water, the energy balance can be divided into the following parts:

(1) The transfer of kinetic energy from the rotation of the solid Earth to the water; this $E_{\text {rot }}$ corresponds to the mean torque.

(2) The loss of kinetic energy of the water by friction on the bottom; this $E_{\text {rel }}$ was previously identified with $\dot{E}_{\text {rot }}$.

(3) The loss of kinetic energy by inner friction in the water, $E_{h}$.

(4) The transfer of kinetic energy from the Moon to the water by tidal forces, $E_{t}$.

One sees that, in the case of the energy, the calculation of one term is not sufficient. Under the assumption that the tidal friction of the hydrosphere is responsible for the astronomical effects, so that each part of the dissipation has the appropriate sign, the loss of rotational energy of the earth must maintain both, the frictional losses and the gain of orbital energy of the Moon. Then the dissipation $E_{\mathrm{rel}}$ is a lower limit for $E_{\mathrm{rot}}$. Under the further assumption that the eccentricity of the Moon's orbit does not change, we can say that the frictional losses will contain the largest part of the dissipation. However, we intend to attack the problem without such assumptions and to calculate all the terms.

Our computations for a model of the Earth are generally restricted to the $M_{2}$-tide and therefore our results correspond to an equatorial 'Moon,' or , in other words, to a planar model with only one significant component of the angular momentum. But within this framework, we will give, at the anticipated end of our computation, the change of angular momentum and the change of the orbital energy of the Moon. This means that the changes of the mean motion and of the eccentricity are determined separately.

Now, after these principal remarks and the outline of our aims, numerical results for specific seas are given. The progress of our work has been hindered severely by the fact that the essential sums for the balances consist of terms of opposite sign, which are orders of magnitude greater than the net effect. Since the errors of the numerical solutions of the hydrodynamical equations have an unknown behaviour, we are not sure, in all cases, about the significance of our net results. 
Our numerical investigations are based on the hydrodynamical-numerical method developed by $\mathbf{W}$. Hansen at the Oceanographic Institute of Hamburg University.

In the following paragraphs, numerical examples are given for the models of the $M_{2}$-tide in the World Ocean, the North Sea and the Bering Sea. Thereby, in each case the loss of kinetic energy by bottom friction, $E_{\text {rel }}$, and the more interesting transfer of kinetic energy from the rotation of the solid earth to the water, $E_{\text {rot }}$, are computed. In this connection, the corresponding torques and the angular momenta are also obtained.

Firstly, let us consider the World Ocean. Our studies are based for technical reasons still on a previously used $10^{\circ}$-model of Zahel.

The loss of kinetic energy by friction at the bottom, $\dot{E}_{\text {rel }}$, integrated over the whole world ocean has phases of stronger and weaker energy dissipation. The mean value over one tidal period is

$$
\left\langle\dot{E}_{\mathrm{rel}}\right\rangle=0.20 \cdot 10^{18} \mathrm{erg} / \mathrm{s} .
$$

This value is small compared with those computed before (Jeffreys 11, Munk $10 \times 10^{18} \mathrm{erg} / \mathrm{s}$ ). One should, however, notice the rough mesh size of the model, which practically does not take into account the influence of shelf areas. And it is a wellknown fact that the main energy dissipation due to bottom friction takes place within the shallow adjacent seas.

If we restrict our attention only to the $z$-component of the torque we can see that there occur - with respect to the Earth's rotation - decelerating and accelerating phases within one tidal period. It is very important that after integration over one tidal period a nonvanishing effect remains. Thus, averaging over time, a permanent torque acts and angular momentum is transferred. This torque, owing to the negative sign, decelerates the Earth.

The $\dot{E}_{\text {rot }}$ obtained (which has to be compared with the astronomical value) is

$$
\left\langle\dot{E}_{\text {rot }}\right\rangle=11 \cdot 10^{18} \mathrm{erg} / \mathrm{s} .
$$

We shall now consider the North Sea, a shallow adjacent sea with strong tides.

The loss of kinetic energy by bottom friction, $\dot{E}_{\text {rel }}$, integrated over the whole North Sea and over one tidal period is

$$
\left\langle\dot{E}_{\mathrm{rel}}\right\rangle=0.41 \cdot 10^{18} \mathrm{erg} / \mathrm{s} \text {. }
$$

This amount is in a good agreement with the value given by Jeffreys $\left(0.36 \cdot 10^{18}\right.$ erg/s).

It was found that the mean transferred torque has a positive sign. Consequently, the tides of the North Sea cause an accelerating effect on the rotation of the Earth! To the best of our knowledge, such a possibility has not been considered as yet.

An explanation for this can be given in terms of the residual currents of the North Sea. This stream pattern which is obtained by averaging the tidal streams over one tidal period, exists permanently. It will be explained by the nonlinear behaviour of motions in shallow water areas which leads to nonharmonic tidal curves with non- 
vanishing mean values with respect to time. In the case of the North Sea, the most evident feature of the residual current system is a broad central stream with a dominating east-directed component, that is, a component in the direction of the Earth's rotation.

Also the geographical distribution of the torques averaged over one tidal period is extremely nonhomogeneous.

For the transfer of kinetic energy from the water to the solid Earth it was found

$$
\left\langle E_{\text {rot }}\right\rangle=-2.4 \cdot 10^{18} \mathrm{erg} / \mathrm{s} .
$$

Finally, the Bering Sea should be considered. This area has played an important role in former investigations.

Figure 1 shows $E_{\text {rel }}$, depending on time. The mean value is

$$
\left\langle\dot{E}_{\mathrm{rel}}\right\rangle=0.28 \cdot 10^{18} \mathrm{erg} / \mathrm{s} \text {. }
$$

which is in good agreement with the result of Munk $\left(0.24 \cdot 10^{18} \mathrm{erg} / \mathrm{s}\right)$.

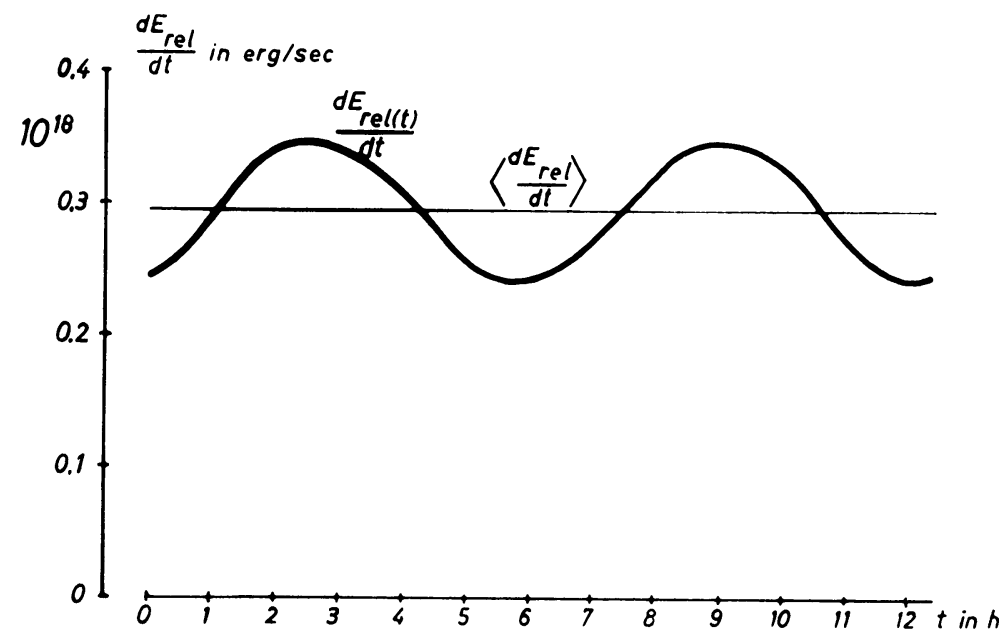

Fig. 1.

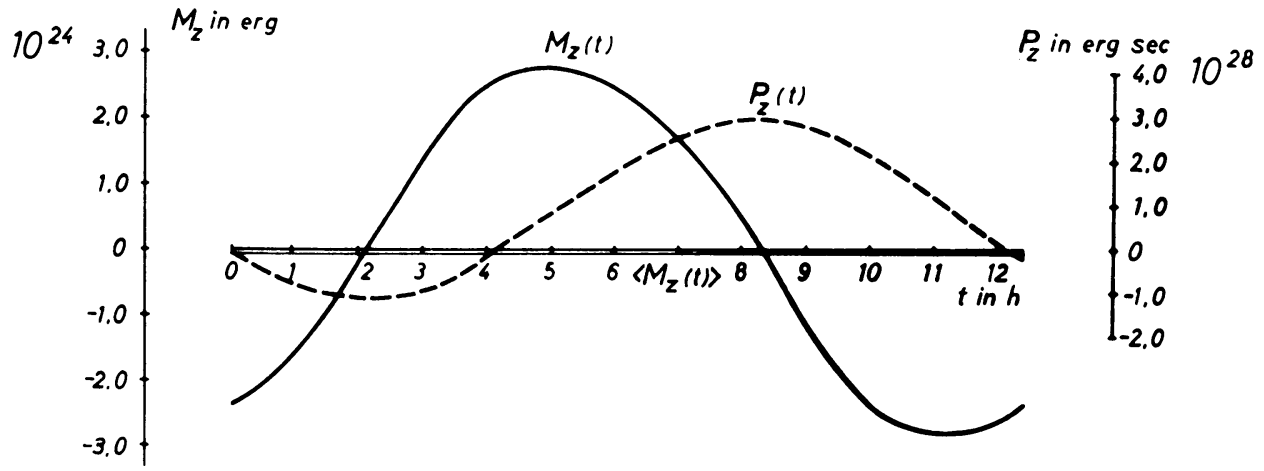

Fig. 2 
In Figure 2 the torque due to tidal friction in the Bering Sea is presented. A negative net effect results, corresponding to a decelerating contribution to the earth's rotation. For $E_{\text {rot }}$ we have obtained

$$
\left\langle E_{\text {rot }}\right\rangle=0.80 \cdot 10^{18} \mathrm{erg} / \mathrm{s} \text {. }
$$

Our models, certainly, should be improved in the future. Especially, we are planning to use a new model of the world ocean (presently with a mesh size of $4^{\circ}$ ) with a refined grid in the shelf areas. Furthermore, also the other energy terms mentioned above should be calculated in order to get a global energy balance. Finally, it is intended to reconstruct the tides for earlier stages of the earth's history.

\section{Reference}

Brosche, P. and Sündermann, J.: 1971, Pure Appl. Geophys. 86, 95.

\section{DISCUSSION}

N. Pariisky: The calculation is right, but the terminology is not: $E$ - is not dissipation, but a change of rotation energy corresponding to the computed torque of the frictional forces.

H. Jeffreys: I have not understood the paper, but I should remark that the dissipation in a particular sea affects the phase of the tide in the whole ocean, and the attraction of the Moon on the whole ocean is what determines the rate of change of the angular momentum.

$P$. Brosche: The results of our model calculations are thought to be the first constituents of the final balance over all seas. 